\title{
Theoretical Evidence for the Distance-Dependent Photoinduced Electron Transfer of Porphyrin-Oligothiophene-Fullerene Triads
}

\author{
Shan Zhang, ${ }^{1}$ Yuanzuo Li, ${ }^{2}$ Jing Liu, ${ }^{1}$ Meiyu Zhao, ${ }^{3}$ Yueyi Han, ${ }^{1}$ \\ Yong Ding, ${ }^{1}$ Peng Song, ${ }^{1}$ and Fengcai $\mathrm{Ma}^{1}$ \\ ${ }^{1}$ Department of Physics, Liaoning University, Shenyang 110036, China \\ ${ }^{2}$ College of Science, Northeast Forestry University, Harbin 150040, China \\ ${ }^{3}$ Institute of Theoretical Simulation Chemistry, Academy of Fundamental and Interdisciplinary Sciences, \\ Harbin Institute of Technology, Harbin 150080, China \\ Correspondence should be addressed to Yuanzuo Li, yuanzuo.li@yahoo.com.cn and Peng Song, songpeng@lnu.edu.cn
}

Received 17 August 2012; Revised 22 October 2012; Accepted 12 November 2012

Academic Editor: Ching-Song Jwo

Copyright (๑) 2012 Shan Zhang et al. This is an open access article distributed under the Creative Commons Attribution License, which permits unrestricted use, distribution, and reproduction in any medium, provided the original work is properly cited.

\begin{abstract}
The ground and excited state properties of $n$ T-C60 dyads and Por- $n$ T-C60 triads $(n=4,8$, and 12$)$ have been theoretically investigated by using the time-dependent density functional theory together with a set of extensive multidimensional visualization techniques. The results reveal that the length of the $n \mathrm{~T}$ moiety strongly influences the charge transfer characters of these systems. The charge transfer ability is largely strengthened by introducing the porphyrin group and decreases with the length of the $n \mathrm{~T}$ moiety. Also the adjustment of the electron transport mode of Por- $n$ T-C60 triads by the length of the $n$ T moiety was visualized. It is found that Por*-4T-C60 shows predominantly the energy transfer process generating Por-4T- ${ }^{1}$ C60* but the charge transfer becomes predominant for other triads, such as the direct formation of Por ${ }^{*+}-12 \mathrm{~T}-\mathrm{C} 60^{\circ-}$ via Por*-12T-C60. Furthermore, the process of Por $^{\bullet-}-8 \mathrm{~T}^{\bullet+}-\mathrm{C} 60 \rightarrow$ Por- $8 \mathrm{~T}^{\bullet+}-\mathrm{C} 60^{\bullet-}$ via Por* $-8 \mathrm{~T}-\mathrm{C} 60$ has been proved to be possible. Finally, the energetically most stable final charge transfer excited state is confirmed to be Por- $\mathrm{nT}^{\bullet+}{ }_{-} \mathrm{C} 60^{\bullet-}$.
\end{abstract}

\section{Introduction}

Conjugated nanoscale molecule has attracted a lot of attention for its potential application in molecular electronic devices, such as organic electroluminescent devices, photovoltaic cells, field-effect transistors, nonlinear optics and electrical conductors $[1,2]$ (and references therein). Photoinduced electron transfer (PET), as a fundamental process, has been proved to play a key role in determining the efficiency of the photoelectric materials [3-5]. Recently, the researchers have focused on the mechanism of PET in organic photoconversion systems with highly efficient charge transfer (CT) and slow charge recombination (CR) [6-8], respectively. The kinetics of these two processes, namely, charge transfer and/or charge recombination, are reflected by the electron transfer rate constant [8]:

$$
k_{\mathrm{ET}}=k_{0} \exp \left(-\beta R_{\mathrm{DA}}\right) \text {, }
$$

where $k_{0}$ is a kinetic prefactor/preexponential factor, $R_{\mathrm{DA}}$ represents the donor-acceptor distance, and $\beta$ is the attenuation factor or dumping factor.

As known, electron and energy transfer reactions in covalently linked donor-bridge-acceptor (D-B-A) assemblies are strongly dependent, not only on the redox properties $\mathrm{D}$ and $\mathrm{A}$ but also on the D-A distance and the electronic structure of the bridge [7]. Visible light excitation, for instance, may result in a charge transfer mediated by the bridge from the photoexcited donor to the acceptor or from the donor to the photoexcited acceptor. According to the remarkable electronic and optical characteristics [9], such as the ready accessibility, structural modifications, highly delocalized $\pi$ conjugation, low oxidation potentials, environmental stability, and well-defined oligothiophenes ( $n \mathrm{Ts}$ ) have emerged as one of the well-investigated oligomers of $\pi$-conjugated polymers [10-12]. The $n \mathrm{~T}$ moiety is assumed to mediate charge transfer between donor and acceptor and 
has been expected to serve as molecular wires for electron transfer and energy transfer in solar cell devices [13-15]. It is known that porphyrins and fullerenes are some of the most ideal efficient electron-donor and acceptor materials for conjugated oligomers and polymers in photovoltaic devices due to their unique structural and electronic properties [11]. The molecular architectures of D-B-A systems formed by porphyrins and fullerenes linked by various bridging groups have been widely studied, and the PET of porphyrin-Bfullerene-conjugated systems proceeded through bridging states as either real or virtual intermediates [15]. Since Ikemoto et al. synthesized the molecules that incorporate a porphyrin as donor and fullerene as acceptor unit which are bridged by quater-, octi-, and dodecithiophenes [9], the porphyrin- $n$ Ts-fullerene triads have been extensively studied as the systems that are widely used in the photoactive layer of solar cell. To investigate the PET mechanism of these triads, the PET processes of porphyrin- $n$ T-C60 linked triads ( $n=4,8$, and 12), which were designed to reveal the function of $n \mathrm{~T}$ as a molecular wire, have been investigated by time-resolved fluorescence and absorption spectroscopic methods in the visible and near-IR regions [2, 16, 17]. The most energetically stable final CS state was confirmed to be porphyrin- $n \mathrm{~T}^{\bullet+}-\mathrm{C} 60^{\bullet-}$, which was produced by the hole-shift process from porphyrin ${ }^{-+}-n \mathrm{~T}-\mathrm{C} 60^{\bullet-}$. The rate constant for the CS process from porphyrin ${ }^{*}-n \mathrm{~T}-\mathrm{C} 60$ to porphyrin $^{\bullet+}-n \mathrm{~T}-\mathrm{C} 60^{\circ-}$ decreased with the length of the $n \mathrm{~T}$ moiety, indicating that the $n \mathrm{~T}$ moiety acts as a molecular wire. And the excited dynamics of these systems in various polar solvents have been investigated. Also, the effects of length of oligothiophene on the CS and CR processes were discussed on the basis of the free energy changes $[16,17]$.

On the other hand, theoretically, the PET in porphyrin4T-fullerene triad has been investigated by using quantum chemistry method as well as the $2 \mathrm{D}$ and $3 \mathrm{D}$ real space analysis methods [15]. The theoretical analysis revealed that there are two charge transfer (CT) mechanisms and the weak distance dependence of the oligothiophene spacer is benefitted from the superexchange mechanism. As known, ultrafast charge transfer from the porphyrin to the fullerene moiety takes place upon the photoexcitation of these triads, following which the long-lived charge-separated states are generated. And the length of oligothiophenes is believed to be one important parameter to influence the efficiency of these processes. So theoretical investigation of the effect of the length of $n \mathrm{~T}$ and introducing the porphyrin (donor) into the system is another important way to study the PET process of the conjugated nanoscale molecule systems, because darkness still covers the nature of it. In this paper, the PET in oligothiophene-C60 ( $n \mathrm{~T}-\mathrm{C} 60)$ dyads and porphyrinoligothiophene-C60 (Por- $n$ T-C60) triads was theoretically investigated, which reveals that the ability of charge transfer of the Por- $n$ T-C60 triads, as a D-B-A system, is much stronger than that of the $n \mathrm{~T}-\mathrm{C} 60$ dyads, as a D-A system. And the ground and excited states properties of these dyads and triads are strongly influenced by the length of the $n \mathrm{~T}$ moieties. Also the adjustment of the electron transport mode of Por- $n$ T-C60 systems by the length of the $n \mathrm{~T}$ moieties was further visualized.

\section{Method}

All of quantum chemical calculations were done with Gaussian 09 software [18]. The ground state geometries of the oligothiophene-fullerene dyads and porphyrinoligothiophene-fullerene triads were optimized using the density function theory (DFT) with B3LYP functional and 3$21 \mathrm{G}$ basis set. Partial density of states (PDOS) was visualized with GaussSum software. The molecular structures of them can be seen from Figure 1 . In the calculations, $\mathrm{R}=\mathrm{C}_{6} \mathrm{H}_{13}$ (the side chain of oligothiophene) was repacked by $\mathrm{H}$, because they were not the direct electronic structure of the conjugated backbone and merely aid in improving solubility without affecting electronic properties [19]. Electronic transitions of $n$ T-C60 and Por- $n$ T-C60 and their donor/acceptor part in optical absorption were performed with timedependent DFT (TD-DFT) [20], B3LYP functional, and 6$31 \mathrm{G}(\mathrm{D})$ basis set, respectively.

The two-dimensional (2D) real space analysis (transition density matrix) and three-dimensional (3D) real space analysis (charge difference density) were described in [2123]. In the $2 \mathrm{D}$ site representation, photoexcitation creates an electron-hole pair or an exciton by moving an electron from an occupied orbital to an unoccupied orbital [22]. Each element of the transition density matrix reflects the dynamics of this exciton projected on a pair of atomic orbitals given by its indices, which gives the probability of finding one charged particle on site $x$ and the second one on site $y$. And the amount of the charged particles reflects the strength of the coherence between the donor and acceptor, which is defined by different color of the element. The 3D cube representation has been used to analyze the charge and energy transfer in conjugated polymers $[19,21-25]$, because the charge difference density (CDD) determines the orientation and results of the charge and energy transfer in molecular and molecular-metal systems.

\section{Results and Discussions}

3.1. Ground State Properties. On the basis of the optimized ground state geometries of $n \mathrm{~T}-\mathrm{C} 60$ (D-A systems) and Por$n$ T-C60 (D-B-A systems), the partial densities of states (PDOS) were calculated and are presented in Figure 2. With the PDOS, we can find the percent contribution of a group to each molecular orbital. As seen from Figures 2(a), 2(c), and 2(e), for $n$ T-C60 ( $n=4,8$, and 12), the complete charge densities of the higher HOMOs are found on the thiophene groups and the charge densities of the lower LUMOs on the C60 group, respectively. This indicates that the transition from these HOMOs to LUMOs of oligothiophene-fullerene systems should be followed by the charge transfers from thiophene to fullerene group. When the porphyrin is introduced into the system, abbreviated as Por- $n$ T-C60 in this paper, the contributions of charge density distribution to the higher HOMOs are originated from both the porphyrin and the thiophene groups (see Figures 2(b), 2(d) and 2(f)). And the percent contribution of the porphyrin group decreases with the length of the $n$ T moiety. Due to the fact that porphyrin group acts as the electron 


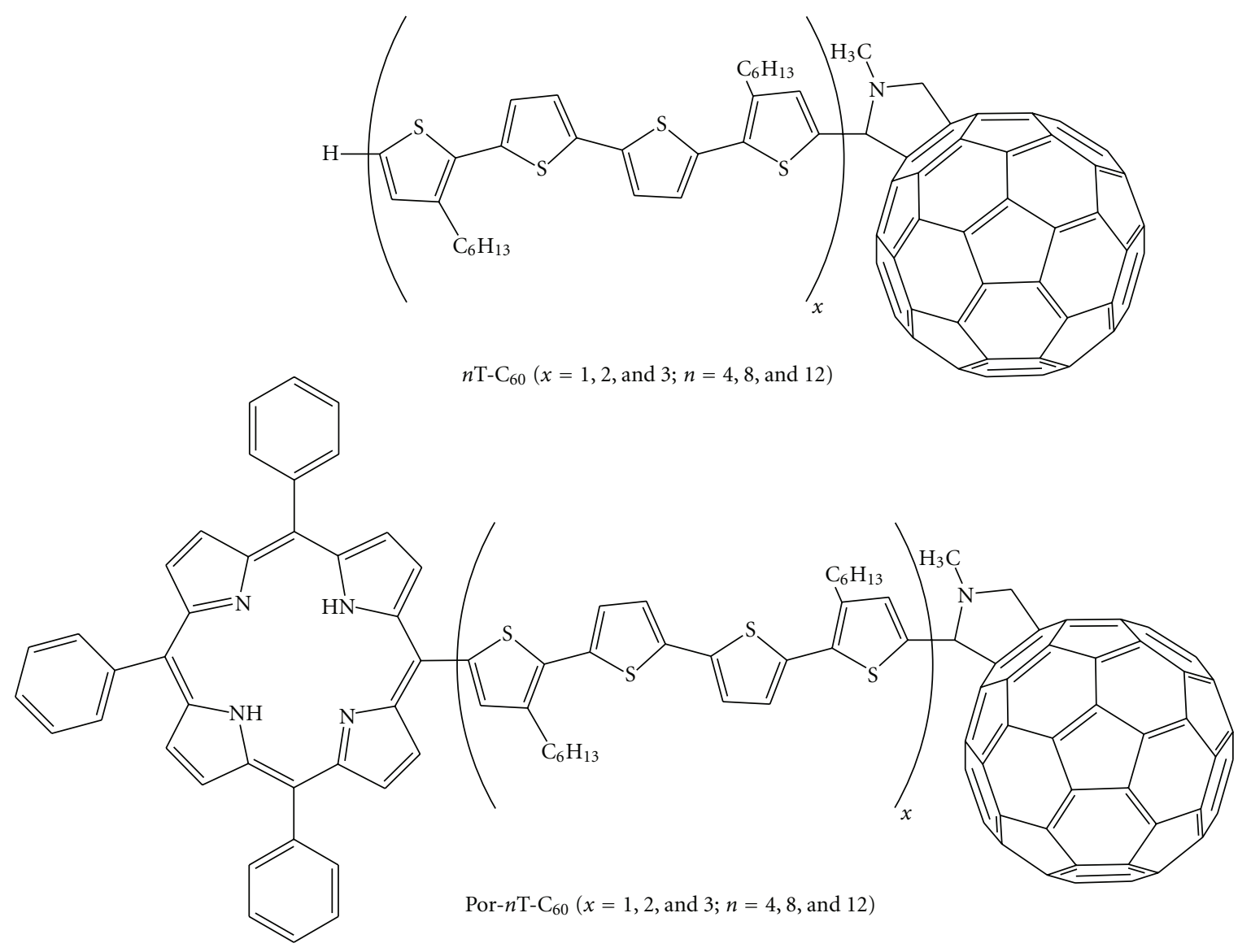

Figure 1: Molecular structures of $n$ T-C60 dyads and Por- $n$ T-C60 triads $(n=4,8$, and 12).

donor of these systems, the small charge density distribution on it in the ground state will result in the decrease of charge transfer from donor to acceptor and the formation of the charge transfer excited state upon the photoexcitation. This is in accordance with the experimental results [2] and will be further investigated in the next section. It should be noted that the percent contribution of charge density distribution to the lower LUMOs molecular orbital is insensitive to the porphyrin group, and for all Por- $n \mathrm{~T}$-C60 systems the charge densities of the lower LUMOs are still completely distributed on the C60 group.

To further investigate the charge distribution on the oligothiophene, which is believed to be adjusted by the length of the thiophene unit and affected by the charge transfer process of these systems, the Mulliken charges on different thiophene moiety are shown in Figure 3, also based on their optimized ground state properties. By comparing Figures 3(a)-3(c), some meaningful conclusion can be obtained. In detail, the Mulliken charge distribution is little influenced by increasing the length of the $n \mathrm{~T}$ moiety but largely affected by introducing the porphyrin group. For $n \mathrm{~T}-\mathrm{C} 60$ dyads, the maximum distribution of Mulliken charges (with positive character) locates on the thiophene moiety, which is adjacent to C60 group. however, for Por- $n$ T-C60 triads, the Mulliken charges almost distribute on the two outer thiophene units and the negative charges on the thiophene moiety, neighbored to the porphyrin group, are much larger than the positive charges on the thiophene unit, which is adjacent to C60. These results together with the further analysis of the excited properties of these D-A and D-B-A systems are expected to give the direct visual evidence for the adjustment of the ICT character by the length of bridge and the introduction of the porphyrin group as the electron donor part.

3.2. Excited State Properties of $n T-C 60$ Dyads. Upon photoexcitation to the excited state, some of the excited state properties of these fullerene-based molecular materials are changed in comparison with those in the ground state. Table 1 gives the excited state properties of these fullerene derivatives, including the selected electronic transition energies, the corresponding oscillator strengths, main compositions, and CI coefficients. For all these fullerene derivatives, the first excited state $\left(\mathrm{S}_{0} \rightarrow \mathrm{S}_{1}\right.$ and abbreviated as $\left.\mathrm{S}_{1}\right)$ mainly originates from the transition from HOMO to LUMO molecular orbits. According to the above PDOS analysis, the electronic transitions involving these orbits are intramolecular charge transfer excited states. To further investigate the change of the static charge distribution upon photoexcitation, the electron-hole coherence and the charge difference densities (CDD) of these electronic transitions 

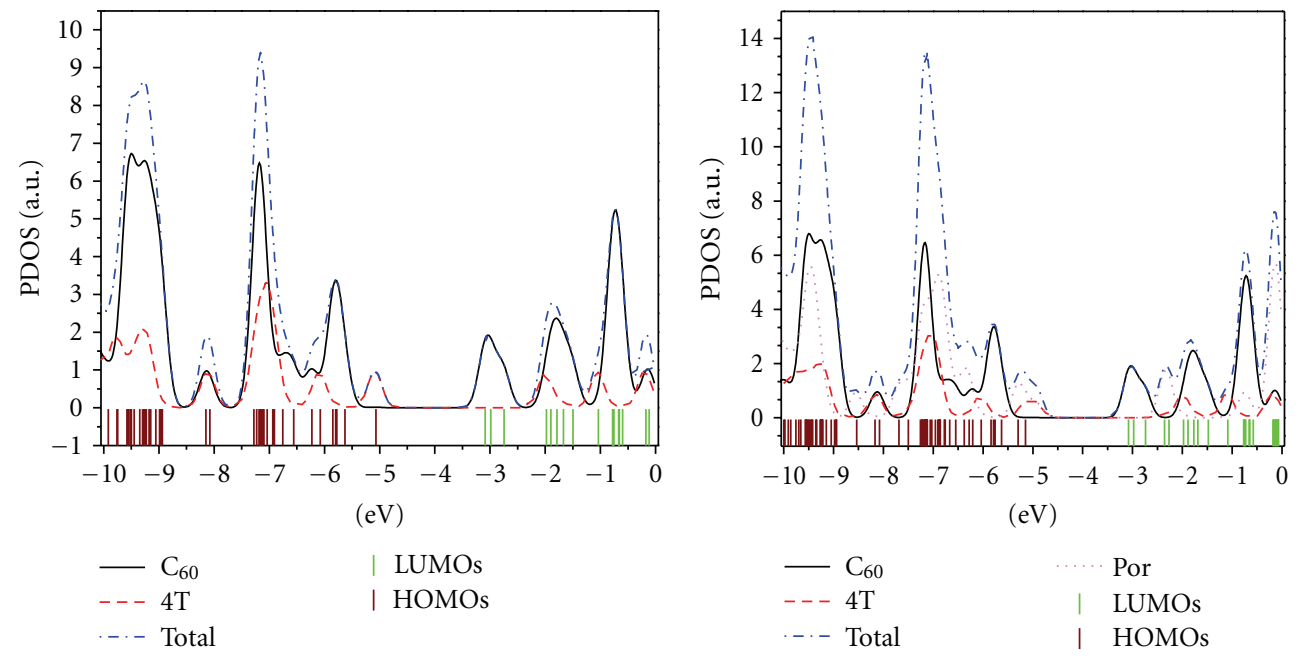

(a)

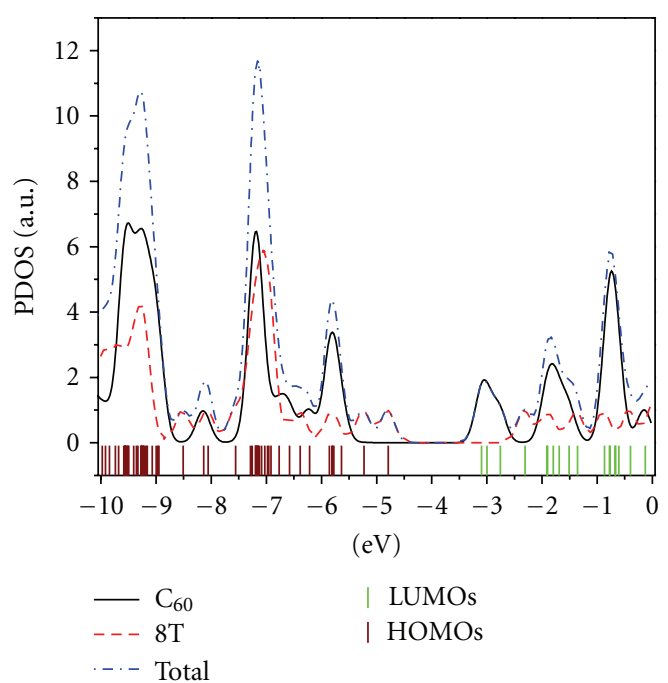

(c)

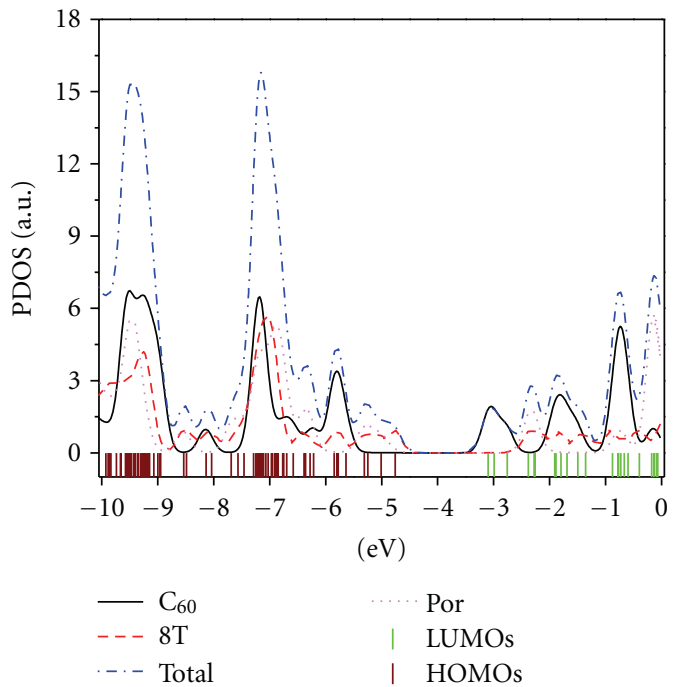

(d)

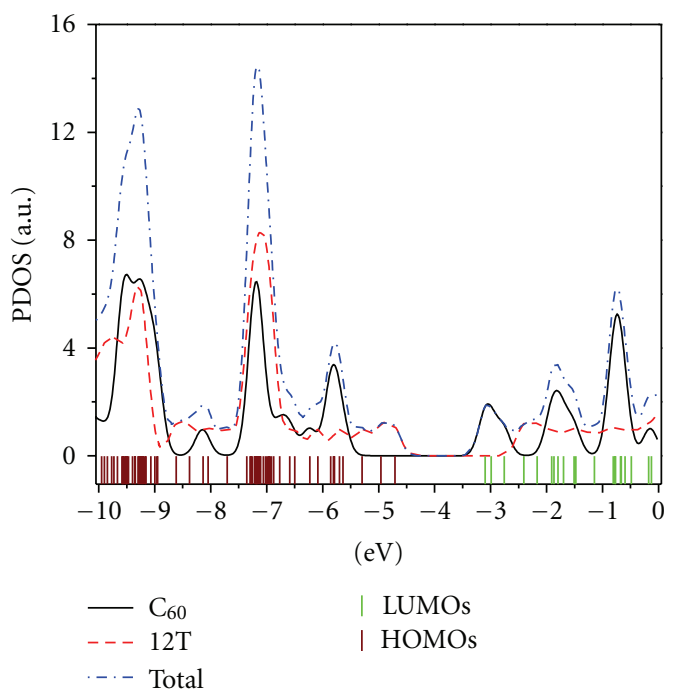

(e)

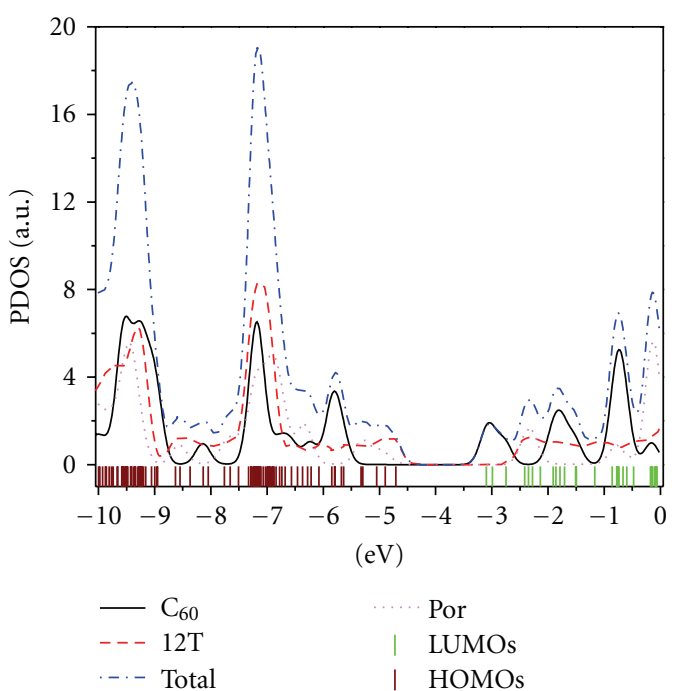

(f)

Figure 2: Partial density of state (PDOS) of $n$ T-C60 ( $n=4,8$, and 12 are in accordance with (a), (c), and (e), resp.) and of Por- $n$ T-C60 $(n=4,8$, and 12 are in accordance with (b), (d), and (f), resp.). 

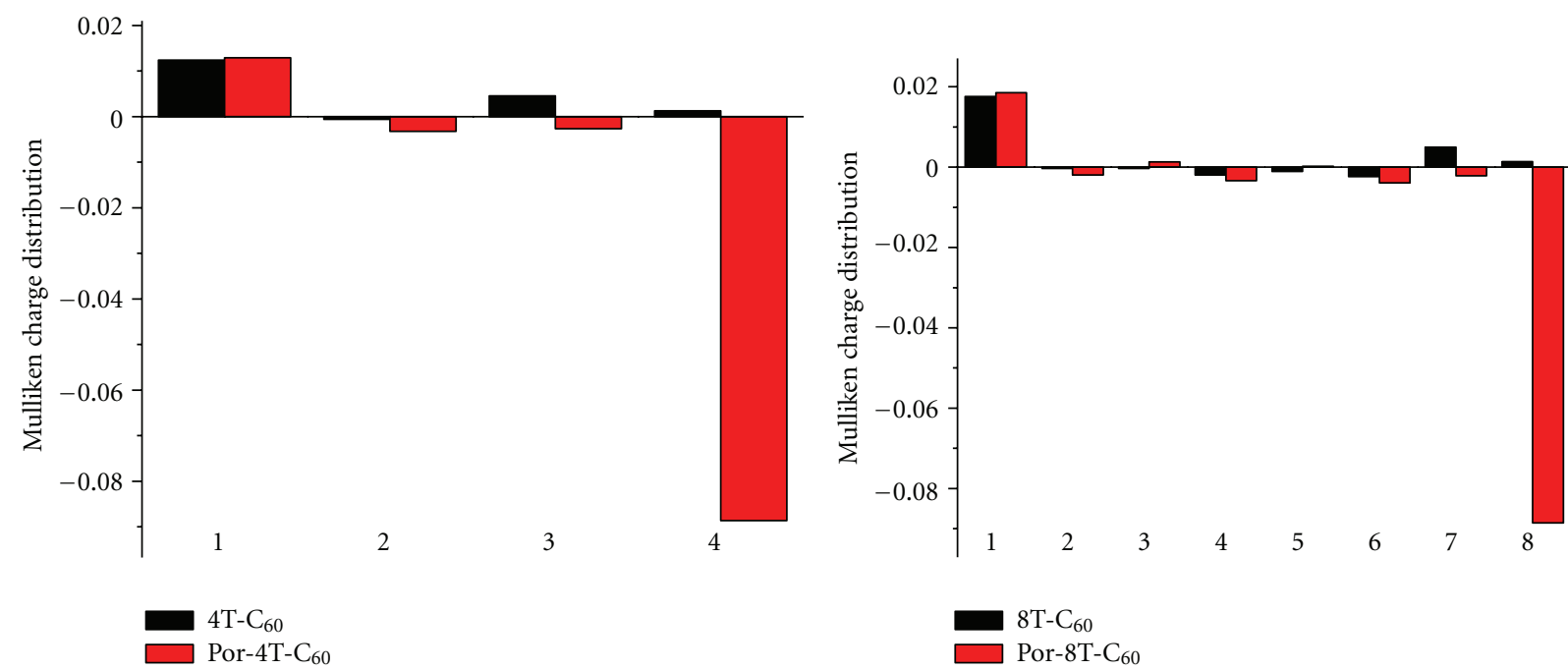

(a)

(b)

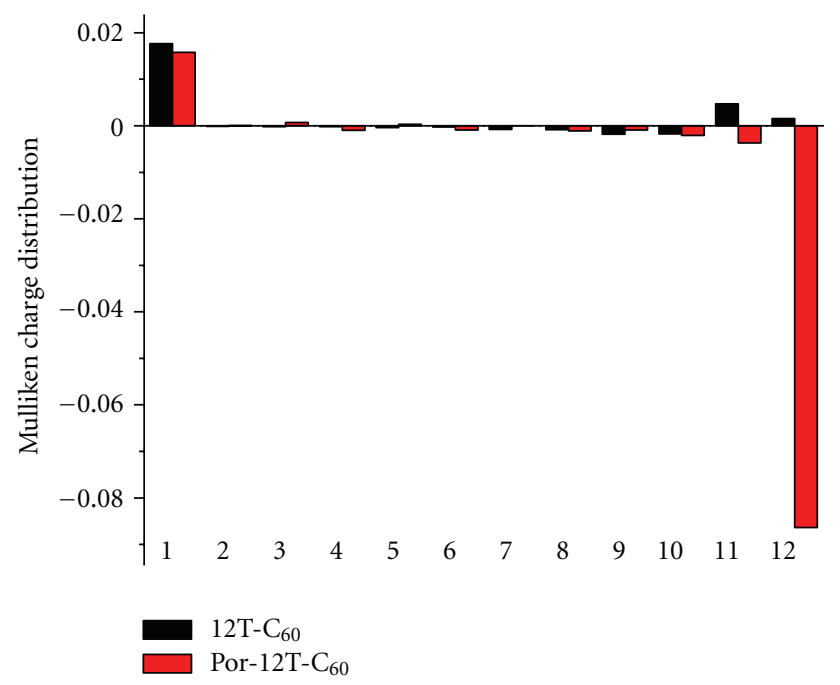

(c)

FIgure 3: The distribution of Mulliken charges on the different thiophene moieties of $n$ T-C60 dyads and Por- $n$ T-C60 triads. The numerals 1-12 stand for the different thiophene unit, which is in the order from the thiophene unit, neighbored to the fullerene group, to the unit, neighbored to the porphyrin group.

are calculated and visualized in the Figures 4 and 5. All of these excited state properties were examined with two representations, which classified as intramolecular charge transfer (ICT) or local excited (LE) transitions (see Table 1). From the 2D contour plots (see Figure 4) of transition density matrix for $\mathrm{S}_{1}$ of $n \mathrm{~T}-\mathrm{C} 60$, we can see that the electron hole coheres between fullerene and thiophene (shown with yellow color), and with increasing the length of the $n$ T moiety the strength of coherence decreases obviously. After analyzing their corresponding CDD (shown in Figure 5), we can clearly identify that the electron transfers from thiophene to fullerene, because the excited electrons and holes completely reside on the fullerene and thiophene groups, respectively. Also the change of the static charge distribution caused by the photoexcitation is significantly influenced by the length of the $n \mathrm{~T}$ moiety. In other words, different thiophene unit in $n \mathrm{~T}$-C60 dyad has different contribution to the transition when the length of the $n$ T moiety is changed. In detail, when $n=4$, the excited holes are delocalized on all these four thiophene units; but when the length of $n \mathrm{~T}$ increases to 8 and 12 units, the excited holes are almost distributed on the center thiophene units, which indicates that the static charge distribution of the outer thiophene units is almost insensitive to the photoexcitation, especially the adjacent unit to the fullerene group. This result is in accordance with the previous researches and again justifies that the excited hole along the $\pi$ conjugation in $8 \mathrm{~T}$ and $12 \mathrm{~T}$ tends to localize in the middle part of the chain more than that in $4 \mathrm{~T}$ because the charge per thiophene ring takes a maximum point in the middle of the chain for $8 \mathrm{~T}$ and $12 \mathrm{~T}[2,26]$. Back to the above Mulliken charge distribution analysis in the ground state, the small distribution of Mulliken charges on the center thiophene 


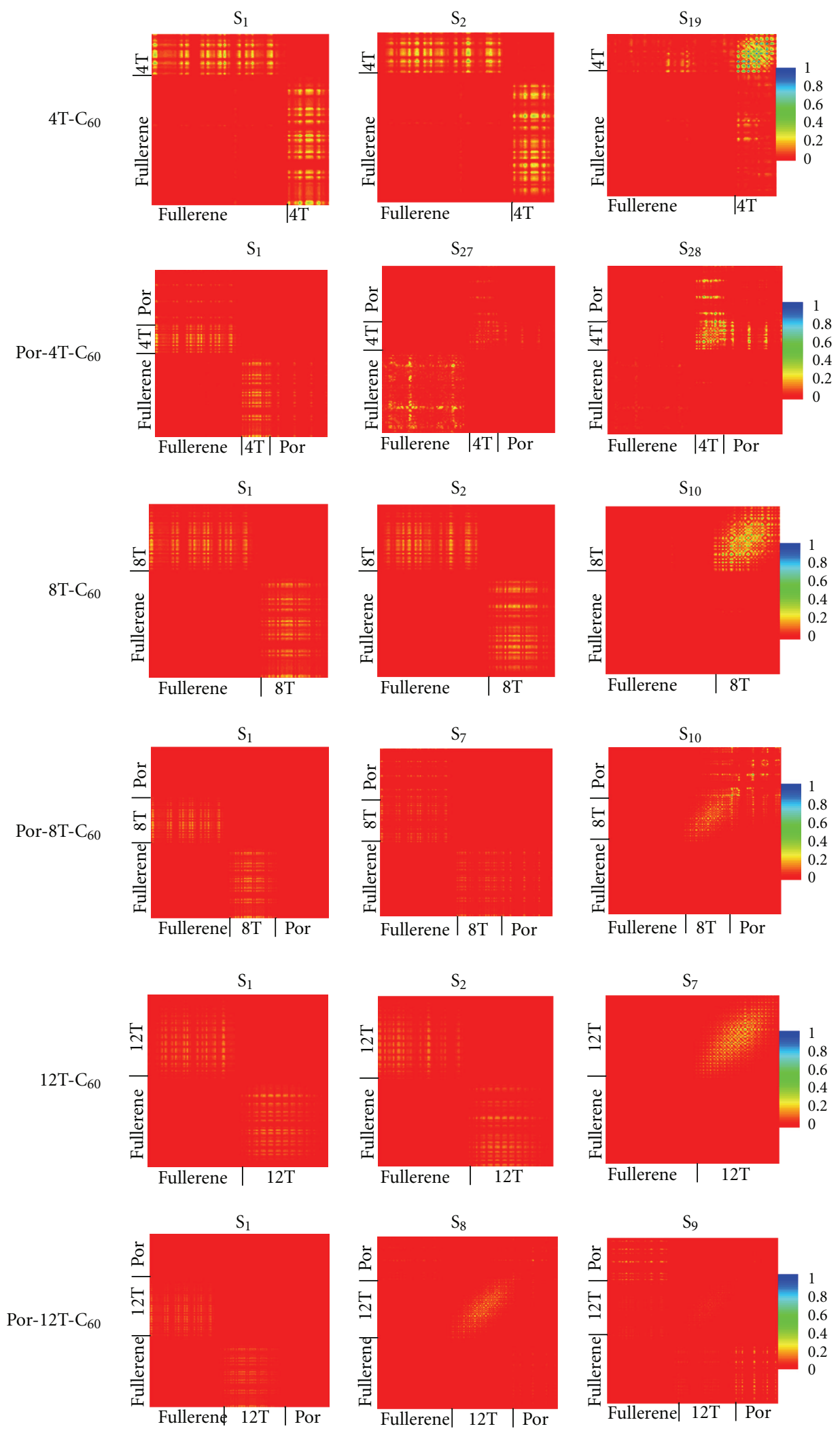

FIgURE 4: The contour plots of transition density matrix of the selected electronic excited states of $n$ T-C60 and Por- $n$ T-C60. The color bar is shown (absolute values of matrix elements, scaled to a maximum value of 1.0), and the electron-hole coherence increases with the increase of absolute values of matrix elements. 


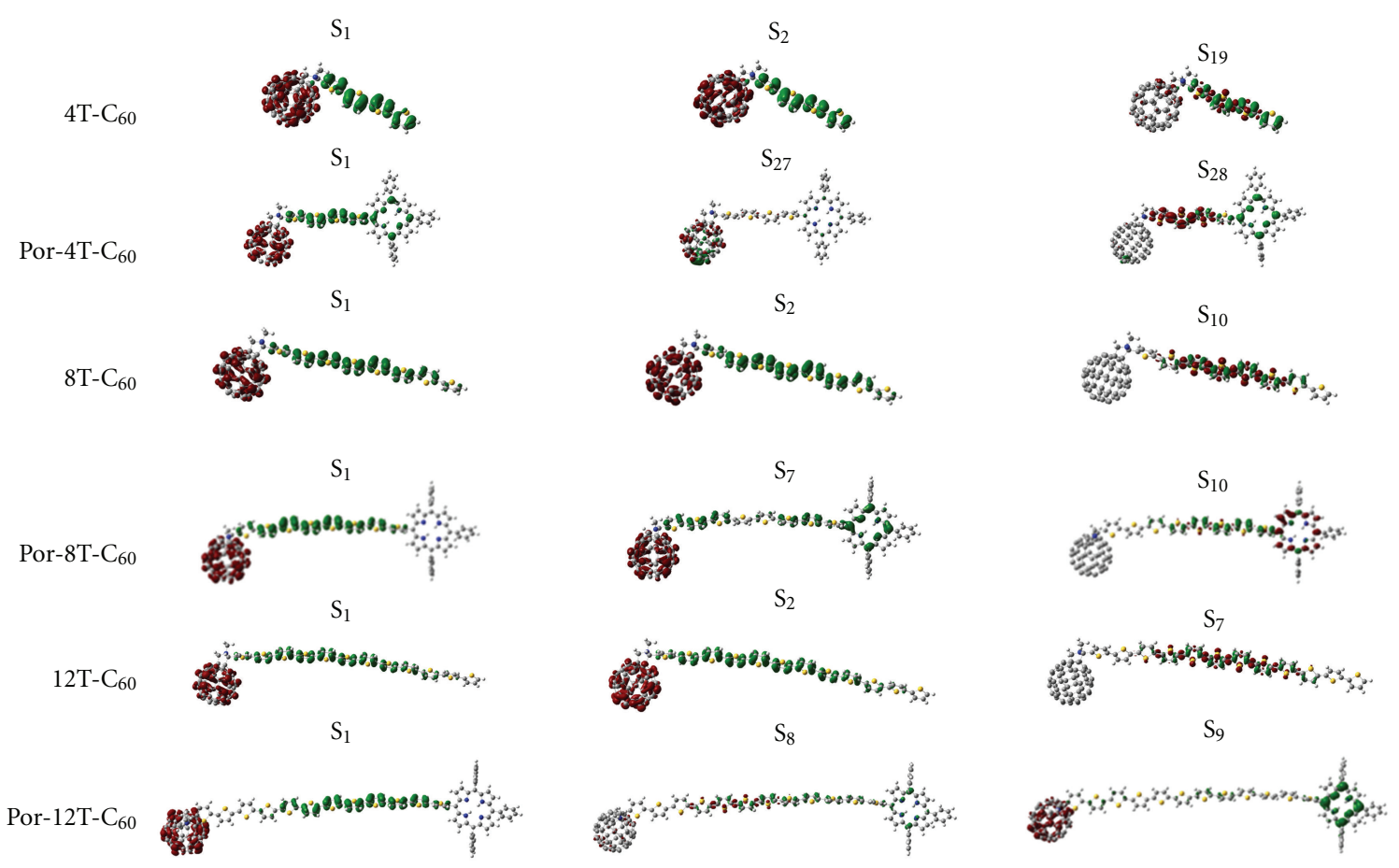

FIgure 5: The charge difference densities (CDD) of the selected electronic excited states of $n$ T-C60 and Por- $n$ T-C60. Green and red colours represent the hole and electron, respectively. The isovalue is $4 \times 10^{-4}$ a.u.

units and the largest Mulliken charge distribution on the thiophene unit, neighbored to the fullerene group, result in the weaker electron-hole coherence between fullerene and thiophenes of $n \mathrm{~T}-\mathrm{C} 60$ dyad by increasing the thiophene units (long bridge). So we can conclude that the charge transfer ability decreases with increasing the length of the $n$ T moiety.

3.3. Function of $n T$ as a Molecular Wire of Por-nT-C60 Triads. When the porphyrin is introduced into the system as the electron donor, the static charge distributions of the thiophene unit of Por- $n$ T-C60 triads behave differently upon photoexcitation to their first excited states. The main difference materializes on the porphyrin neighbored thiophene moiety, which has the largest negative Mulliken charge distribution in the ground state (see Figure 3) and acts as the main contributor for the transition from the ground state to $S_{1}$ of Por- $n$ T-C60 triads (as shown in Figure 5). This informs that the ability of charge transfer of the Por- $n$ T-C60 triads, as a D-B-A system, is much stronger than that of the $n$ T-C60 dyads, as a D-A system. Similar to the $n$ T-C60 dyads, with increasing the length of the $n \mathrm{~T}$ moiety, the account of the excited holes on the thiophene moiety, neighbored to the porphyrin moiety, decreases largely, which suggests that the electron transfer abilities of Por- $n$ T-C60 triads are in the order of $4 \mathrm{~T}>8 \mathrm{~T}>12 \mathrm{~T}$ and the charge density of Por- $4 \mathrm{~T}^{\bullet+}-\mathrm{C} 60^{\bullet-}$ much larger than those in other triads [2]. This result is in accordance with the electron-hole coherence between the thiophene and fullerene groups (as can be seen from Figure 4). It should be noted that, for Por-4T-C60 triad, the weaker electron-hole coherence between the porphyrin and fullerene groups could be even found, because the space distance between them is short enough.

Furthermore, the adjustment of the electron transport mode of Por- $n$ T-C60 systems by the length of the $n$ T moieties can be further visualized. All of the possible energy transfer (EN) and charge transfer (CT) processes of these triads can be summarized as follows:

$$
\begin{aligned}
\text { Por }^{*}-n \mathrm{~T}-\mathrm{C} 60 \stackrel{k_{\mathrm{CT}}+k_{\mathrm{EN}}}{\longrightarrow} & \text { Por }^{\bullet+}-n \mathrm{~T}-\mathrm{C} 60^{\bullet-} \\
& + \text { Por }^{\bullet-}-n \mathrm{~T}^{\bullet+}-\mathrm{C} 60+\text { Por- } n \mathrm{~T}-\mathrm{C} 60^{*} .
\end{aligned}
$$

After photoexcitation of the porphyrin moiety of the Por- $n$ T-C60 triads, the Por* $-n$ T-C60 is produced, following which the different excited state dynamic relaxation processes take place, and controlled by the length of the $n$ T moieties. As can be seen from Table 1, the calculated transition energies of Por- $n$ T-C60 triads with the largest oscillator strengths have been listed, from which the different dynamic processes of these triads via Por* ${ }^{*} n$ T-C60 can be revealed. In the case of Por-4T-C60, the twenty-seventh and twenty-eighth excited states $\left(S_{27}\right.$ and $\left.S_{28}\right)$ were calculated to be the excited state with the larger oscillator strengths. In addition, from the CCD plot of the $S_{27}$ excited state (see Figure 5), the EN process to the $\mathrm{C} 60$ moiety can be well visualized. And this result is in good agreement with the experiment, which has proved that the CS process to Por ${ }^{\bullet+}-4 \mathrm{~T}-\mathrm{C} 60^{\bullet-}$ via Por* $-4 \mathrm{~T}-$ $\mathrm{C} 60$ is not possible because of the positive $\Delta G_{\mathrm{CS}}$ value [2]. So the $4 \mathrm{~T}$ moiety acts as a spacer for EN, because the EN process driven by the Dexter mechanism, which is operative in the short-distance EN process, does not operate to $R_{\mathrm{DA}}=$ 
TABLE 1: Selected electronic transition energies $(\mathrm{eV})$ and the corresponding oscillator strengths $(f)$, main compositions, and CI coefficients of $n$ T-C60 and Por- $n$ T-C60.

\begin{tabular}{|c|c|c|c|c|c|}
\hline Compounds & Excited states & Transition energy $(\mathrm{eV})^{\mathrm{a}}$ & $f^{\mathrm{b}}$ & $\mathrm{CI}^{\mathrm{c}}$ & Excited state property ${ }^{\mathrm{d}}$ \\
\hline \multirow{3}{*}{ 4T-C60 } & $\mathrm{S}_{1}$ & $1.7127(724 \mathrm{~nm})$ & 0.0025 & $0.70104(\mathrm{H} \rightarrow \mathrm{L})$ & ICT \\
\hline & $\mathrm{S}_{2}$ & $1.8154(683 \mathrm{~nm})$ & 0.0002 & $0.70047(\mathrm{H} \rightarrow \mathrm{L}+1)$ & ICT \\
\hline & $\mathrm{S}_{19}$ & $2.8127(441 \mathrm{~nm})$ & 1.2480 & $0.63249(\mathrm{H} \rightarrow \mathrm{L}+3)$ & $\mathrm{LE}(4 \mathrm{~T})$ \\
\hline \multirow{3}{*}{ Por-4T-C60 } & $\mathrm{S}_{1}$ & $1.6171(767 \mathrm{~nm})$ & 0.0001 & $0.67616(\mathrm{H} \rightarrow \mathrm{L})$ & $\mathrm{CT}$ \\
\hline & $\mathrm{S}_{27}$ & $2.6127(475 \mathrm{~nm})$ & 0.2022 & $0.52142(\mathrm{H}-8 \rightarrow \mathrm{L}+1)$ & $\mathrm{LE}(\mathrm{F})$ \\
\hline & $\mathrm{S}_{28}$ & $2.6146(474 \mathrm{~nm})$ & 0.9764 & $0.59820(\mathrm{H} \rightarrow \mathrm{L}+5)$ & $\mathrm{CT}$ \\
\hline \multirow{3}{*}{ 8T-C60 } & $\mathrm{S}_{1}$ & $1.5079(822 \mathrm{~nm})$ & 0.0030 & $0.69760(\mathrm{H} \rightarrow \mathrm{L})$ & ICT \\
\hline & $\mathrm{S}_{2}$ & $1.6129(769 \mathrm{~nm})$ & 0.0003 & $0.69703(\mathrm{H} \rightarrow \mathrm{L}+1)$ & ICT \\
\hline & $\mathrm{S}_{10}$ & $2.1813(568 \mathrm{~nm})$ & 2.9247 & $0.68669(\mathrm{H} \rightarrow \mathrm{L}+3)$ & $\mathrm{LE}(8 \mathrm{~T})$ \\
\hline \multirow{3}{*}{ Por-8T-C60 } & $\mathrm{S}_{1}$ & $1.4949(829 \mathrm{~nm})$ & 0.0031 & $0.69223(\mathrm{H} \rightarrow \mathrm{L})$ & CT \\
\hline & $\mathrm{S}_{7}$ & $1.9918(622 \mathrm{~nm})$ & 0.0092 & $0.62072(\mathrm{H}-2 \rightarrow \mathrm{L})$ & ICT \\
\hline & $\mathrm{S}_{10}$ & $2.0660(600 \mathrm{~nm})$ & 2.2460 & $0.58463(\mathrm{H} \rightarrow \mathrm{L}+3)$ & $\mathrm{CT}$ \\
\hline \multirow{3}{*}{$12 \mathrm{~T}-\mathrm{C} 60$} & $\mathrm{~S}_{1}$ & $1.4746(841 \mathrm{~nm})$ & 0.0025 & $0.68307(\mathrm{H} \rightarrow \mathrm{L})$ & ICT \\
\hline & $\mathrm{S}_{2}$ & $1.5792(785 \mathrm{~nm})$ & 0.0001 & $0.68245(\mathrm{H} \rightarrow \mathrm{L}+1)$ & ICT \\
\hline & $\mathrm{S}_{7}$ & $1.9785(627 \mathrm{~nm})$ & 4.2837 & $0.68219(\mathrm{H} \rightarrow \mathrm{L}+3)$ & LE $(12 \mathrm{~T})$ \\
\hline \multirow{3}{*}{ Por-12T-C60 } & $\mathrm{S}_{1}$ & $1.4899(832 \mathrm{~nm})$ & 0.0002 & $0.67812(\mathrm{H} \rightarrow \mathrm{L})$ & $\mathrm{CT}$ \\
\hline & $\mathrm{S}_{8}$ & $1.9462(637 \mathrm{~nm})$ & 3.7154 & $0.55718(\mathrm{H} \rightarrow \mathrm{L}+3)$ & $\mathrm{CT}$ \\
\hline & $\mathrm{S}_{9}$ & $1.9543(634 \mathrm{~nm})$ & 0.9797 & $0.57073(\mathrm{H}-2 \rightarrow \mathrm{L}+1)$ & $\mathrm{CT}$ \\
\hline
\end{tabular}

${ }^{\mathrm{a}}$ The numbers in parentheses are the transition energy in wavelength. ${ }^{\mathrm{b}}$ Oscillator strength. ${ }^{\mathrm{c}} \mathrm{CI}$ coefficients are in absolute values. $\mathrm{H}$ stands for $\mathrm{HOMO}$, and $\mathrm{L}$ stands for LUMO. ${ }^{\mathrm{d}} \mathrm{F}$ and $n \mathrm{~T}$ in parentheses present that the density is localized on the fullerene and thiophenes units, respectively. ICT presents the complete charge transfer state of $n$ T-C60, and CT presents the partly charge transfer state of Por- $n$ T-C60.

$55.7 \AA$ for Por-12T-C60 [2, 27]. Reasonably, the presence of the stronger electron-hole coherence between the porphyrin and C60 moieties with yellow color in Por-4T-C60 triad in Figure 4 could facilitate its EN process. After EN process, from its CCD plot of the $S_{1}$ excited state in Figure 5, the formation of Por- $4 \mathrm{~T}^{\cdot+}-\mathrm{C} 60^{\circ-}$, following the charge transfer of the $n \mathrm{~T}$ moiety and the ${ }^{1} \mathrm{C} 60^{*}$ moiety, can be easily seen. So the excited state dynamic relaxation process of Por-4T-C60 should be described as Por*-4T-C60 $\rightarrow$ Por-4T- ${ }^{1}$ C60* $\rightarrow$ Por- $4 \mathrm{~T}^{*+}-\mathrm{C}_{60}{ }^{\circ-}$. For Por-12T-C60, the eighth and ninth excited states $\left(S_{8}\right.$ and $\left.S_{9}\right)$, with the largest oscillator strengths, present the obvious charge transfer character because the excited holes and electrons localize on porphyrin and C60 moieties, respectively (see the CCD plots of the $S_{8}$ and $S_{9}$ excited state of Por-12T-C60 in Figure 5). This results in that the direct generation of the CS state, $\mathrm{Por}^{\circ+}-12 \mathrm{~T}$ $\mathrm{C}^{\circ} 0^{\circ-}$, is produced from Por $^{*}-12 \mathrm{~T}-\mathrm{C} 60$ and indicates that the $n \mathrm{~T}$ moieties serve as superior molecular wires allowing an efficient long-range CS process from the Por* moiety to the $\mathrm{C} 60$ moiety through the large $n \mathrm{~T}$ moiety. Similar to Por-4T-C60, the hole-shift process of Por-12T-C60 also takes place, which can be clearly seen from its CCD plot of the $S_{1}$ excited state in Figure 5. And the excited state dynamic relaxation process of Por-12T-C60 should be Por*12T-C60 $\rightarrow$ Por $^{\bullet+}-12 \mathrm{~T}-\mathrm{C} 60^{\circ-} \rightarrow$ Por- $12 \mathrm{~T}^{\bullet+}-\mathrm{C} 60^{\circ-}$. Lastly, some different results of Por-8T-C60, as compared to those of the experiment, were obtained here. From the CCD plot of the $S_{10}, S_{7}$, and $S_{1}$ excited states of Por-8T-C60 in Figure 5, the excited state dynamic relaxation process of Por $^{\bullet-}-8 \mathrm{~T}^{\bullet+}$ C60 $\rightarrow$ Por- $8 \mathrm{~T}^{*+}-\mathrm{C} 60^{\circ-}$ via Por*-8T-C60 is well visualized and proved to be possible. In detail, the charge transfer between porphyrin and $8 \mathrm{~T}$ moieties (the formation of Por ${ }^{\circ-}$ $8 \mathrm{~T}^{*+}-\mathrm{C} 60$ ) takes place. Following which the excited electrons transfer from Por $^{\bullet-}$ to C60 moieties, because the electronacceptor abilities have been proved to be in the order of Por $\approx 8 \mathrm{~T} \ll \mathrm{C} 60$ [2]. At the same time, the hole shifts from Por ${ }^{\bullet+}$ moiety to $8 \mathrm{~T}$ moiety and the excited holes redistribution on the $8 \mathrm{~T}$ take place.

\section{Conclusion}

From the calculating ground state results of $n \mathrm{~T}-\mathrm{C} 60$ dyads and Por- $n$ T-C60 triads, the obvious charge transfer characters of these systems have been presented. The contribution of the porphyrin group to the charge transfer in Por- $n \mathrm{~T}-\mathrm{C} 60$ triads decreases with the length of the $n \mathrm{~T}$ moiety, and the Mulliken charge distribution on different thiophene moiety is largely affected by introducing the porphyrin group. For $n$ T-C60 dyads, the charge transfer ability decreases with increasing the length of the $n \mathrm{~T}$ moiety. The ability of charge transfer of the Por- $n$ T-C60 triads is proved to be much stronger than that of the $n \mathrm{~T}-\mathrm{C} 60$ dyads and be in the order of $4 \mathrm{~T}>8 \mathrm{~T}>12 \mathrm{~T}$. The excited state dynamic relaxation process of Por-4T-C60 and Por-12T-C60 can be described as Por*$4 \mathrm{~T}-\mathrm{C} 60 \rightarrow$ Por- $4 \mathrm{~T}-{ }^{1} \mathrm{C} 60^{*} \rightarrow$ Por- $4 \mathrm{~T}^{*+}-\mathrm{C} 60^{\circ-}$ and Por*-12T$\mathrm{C} 60 \rightarrow$ Por $^{\bullet+}-12 \mathrm{~T}-\mathrm{C} 60^{\circ-} \rightarrow$ Por- $12 \mathrm{~T}^{{ }^{+}-}{ }_{-} \mathrm{C} 60^{\circ-}$, respectively. 


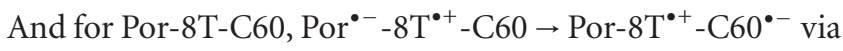
Por*-8T-C60 has been proved to be possible.

\section{Acknowledgments}

This work was supported by the Program of Shenyang Key Laboratory of Optoelectronic Materials and Technology (Grant no. F12-254-1-00), the Fundamental Research Funds for the Central Universities (Grant no. DL12BB19), the National Natural Science Foundation of China (Grants no. 11274149 and 21271095), the Natural Science Foundation of Liaoning Province of China (Grants no. 20111035, 201102080 and 20121032), Liaoning Provincial Department of Education Project (Grant no. L20111003), and the Natural Science Foundation of Liaoning University (Grant no. 2011LDGY10).

\section{References}

[1] M. T. Sun and H. X. Xu, "A novel application of plasmonics: plasmon-driven surface-catalyzed reactions," Small, vol. 8, no. 18, pp. 2777-2786, 2012.

[2] M. T. Sun, Z. L. Zhang, H. R. Zheng, and H. X. Xu, "In-situ plasmon-driven chemical reactions revealed by high vacuum tip-enhanced Raman spectroscopy," Scientific Reports, vol. 2, no. 647, 2012.

[3] T. Nakamura, M. Fujitsuka, Y. Araki et al., "Photoinduced electron transfer in porphyrin-oligothiophene-fullerene linked triads by excitation of a porphyrin moiety," Journal of Physical Chemistry B, vol. 108, no. 30, pp. 10700-10710, 2004.

[4] F. Oswald, D. M. S. Islam, Y. Araki et al., "High effectiveness of oligothienylenevinylene as molecular wires in Zn-porphyrin and C60 connected systems," Chemical Communications, no. 43, pp. 4498-4500, 2007.

[5] T. Vuorinen, K. Kaunisto, V. Chukharev, N. V. Tkachenko, A. Efimov, and H. Lemmetyinen, "Kinetics of photoinduced electron transfer in polythiophene-porphyrin- fullerene molecular films," Journal of Physical Chemistry B, vol. 110, no. 39, pp. 19515-19520, 2006.

[6] A. A. Bakulin, A. Rao, V. G. Pavelyev et al., "The role of driving energy and delocalized states for charge separation in organic semiconductors," Science, vol. 335, no. 6074, pp. 1340-1344, 2012.

[7] B. Albinsson, M. P. Eng, K. Pettersson, and M. U. Winters, "Electron and energy transfer in donor-acceptor systems with conjugated molecular bridges," Physical Chemistry Chemical Physics, vol. 9, no. 44, pp. 5847-5864, 2007.

[8] D. M. Guldi, B. M. Illescas, C. M. Atienza, M. Wielopolski, and N. Martín, "Fullerene for organic electronics," Chemical Society Reviews, vol. 38, no. 6, pp. 1587-1597, 2009.

[9] J. Ikemoto, K. Takimiya, Y. Aso, T. Otsubo, M. Fujitsuka, and O. Ito, "Porphyrin-oligothiophene-fullerene triads as an efficient intramolecular electron-transfer system," Organic Letters, vol. 4, no. 3, pp. 309-311, 2002.

[10] J. Liu, T. Tanaka, K. Sivula, A. P. Alivisatos, and J. M. J. Fréchet, "Employing end-functional polythiophene to control the morphology of nanocrystal-polymer composites in hybrid solar cells," Journal of the American Chemical Society, vol. 126, no. 21 , pp. 6550-6551, 2004.
[11] A. Mishra, C. Q. Ma, and P. Bäuerle, "Functional oligothiophenes: molecular design for multidimensional nanoarchitectures and their applications," Chemical Reviews, vol. 109, no. 3, pp. 1141-1276, 2009.

[12] N. M. Boylea, J. Rochfordb, and M. T. Prycea, "Thienylappended porphyrins: synthesis, photophysical and electrochemical properties, and their applications," Coordination Chemistry Reviews, vol. 254, no. 1-2, pp. 77-102, 2010.

[13] T. Otsubo, Y. Aso, and K. Takimiya, "Functional oligothiophenes as advanced molecular electronic materials," Journal of Materials Chemistry, vol. 12, no. 9, pp. 2565-2575, 2002.

[14] P. A. Van Hal, R. A. J. Janssen, G. Lanzani, G. Cerullo, M. Zavelani-Rossi, and S. De Silvestri, "Full temporal resolution of the two-step photoinduced energy-electron transfer in a fullerene-oligothiophene-fullerene triad using sub-10 fs pump-probe spectroscopy," Chemical Physics Letters, vol. 345, no. 1-2, pp. 33-38, 2001.

[15] M. Sun, P. Song, Y. Chen, and F. Ma, "Intramolecular charge transfer in the porphyrin-oligothiophene-fullerene triad," Chemical Physics Letters, vol. 416, no. 1-3, pp. 94-99, 2005.

[16] M. Fujitsuka, O. Ito, T. Yamashiro, Y. Aso, and T. Otsubo, "Solvent polarity dependence of photoinduced charge separation in a tetrathiophene-ceo dyad studied by pico- and nanosecond laser flash photolysis in the near-IR region," Journal of Physical Chemistry A, vol. 104, no. 21, pp. 4876-4881, 2000.

[17] M. Fujitsuka, K. Matsumoto, O. Ito, T. Yamashiro, Y. Aso, and T. Otsubo, "Pico- and nano-second laser flash photolysis study on photoinduced charge separation in oligothiopheneC60 dyad molecules," Research on Chemical Intermediates, vol. 27, no. 1-2, pp. 73-88, 2001.

[18] M. J. Frisch, G. W. Trucks, H. B. Schlegel et al., Gaussian 09, Revision A.1, Gaussian, Wallingford, UK, 2009.

[19] Y. Z. Li, T. Pullerits, M. Y. Zhao, and M. T. Sun, "Theoretical characterization of the $\mathrm{PC}_{60} \mathrm{BM}$ :PDDTT model for an organic solar cell," The Journal of Physical Chemistry C, vol. 115, no. 44, pp. 21865-21873, 2011.

[20] R. E. Stratmann, G. E. Scuseria, and M. J. Frisch, "An efficient implementation of time-dependent density-functional theory for the calculation of excitation energies of large molecules," Journal of Chemical Physics, vol. 109, no. 19, pp. 8218-8224, 1998.

[21] W. J. D. Beenken and T. Pullerits, "Spectroscopic units in conjugated polymers: a quantum chemically founded concept?" Journal of Physical Chemistry B, vol. 108, no. 20, pp. 61646169, 2004.

[22] S. Tretiak, A. Saxena, R. L. Martin, and A. R. Bishop, "Conformational dynamics of photoexcited conjugated molecules," Physical Review Letters, vol. 89, no. 9, pp. 974021-974024, 2002.

[23] Y. Z. Li, F. C. Ma, B. Dong, J. Li, and M. D. Chen, "Theoretical study of charge transfer mechanism in fullerene-phenylphenothiazine compound: a real-space analysis," Dyes and Pigments, vol. 92, no. 3, pp. 1344-1350, 2012.

[24] J. Rissler, H. Bassler, F. Gebhard, and P. Schwerdtfeger, "Excited states of ladder-type poly-p-phenylene oligomers," Physical Review B, vol. 64, Article ID 045122, 11 pages, 2001.

[25] S. S. Liu, X. M. Zhao, Y. Z. Li, and M. D. Chen, "Density functional theory study on Herzberg-Teller contribution in Raman scattering from 4-aminothiophenol-metal complex and metal-4-aminothiophenol-metal junction," The Journal of Chemical Physics, vol. 130, Article ID 234509, 9 pages, 2009.

[26] V. M. Geskin, A. Dkhissi, and J. L. Brédas, "Oligothiophene radical cations: polaron structure in hybrid DFT and MP2 calculations," International Journal of Quantum Chemistry, vol. 91, no. 3, pp. 350-354, 2003. 
[27] D. L. Dexter, "A theory of sensitized luminescence in solids," The Journal of Chemical Physics, vol. 21, no. 5, pp. 836-850, 1953. 


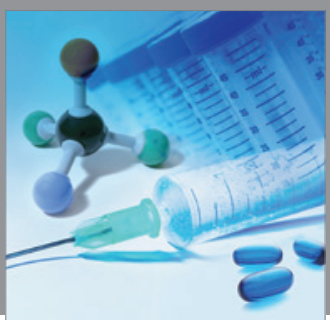

International Journal of

Medicinal Chemistry

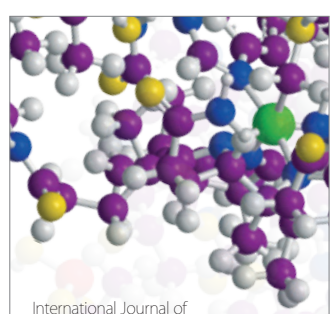

Carbohydrate Chemistry

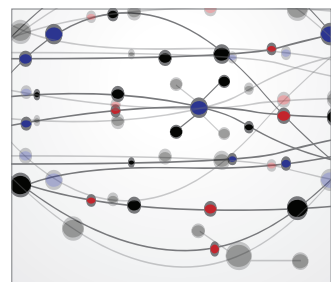

The Scientific World Journal
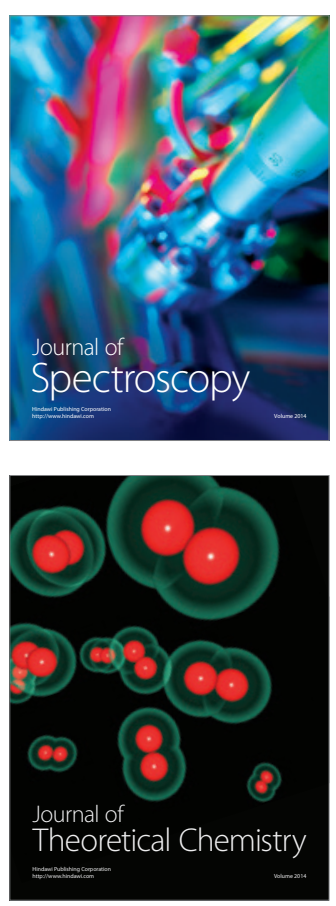
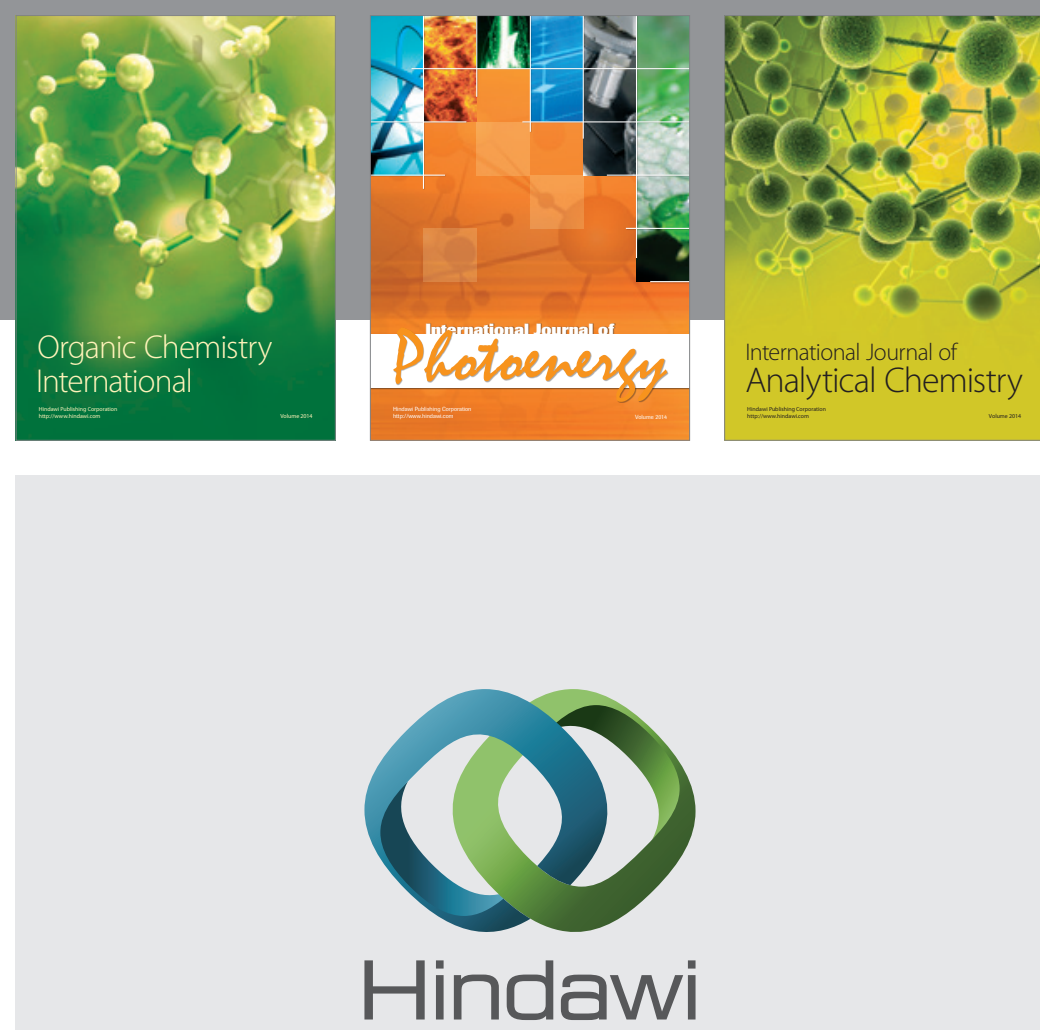

Submit your manuscripts at

http://www.hindawi.com
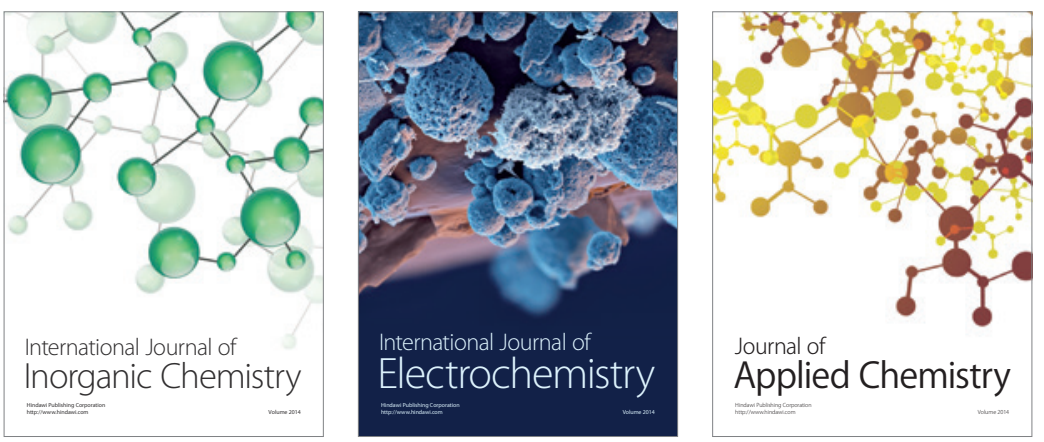

Journal of

Applied Chemistry
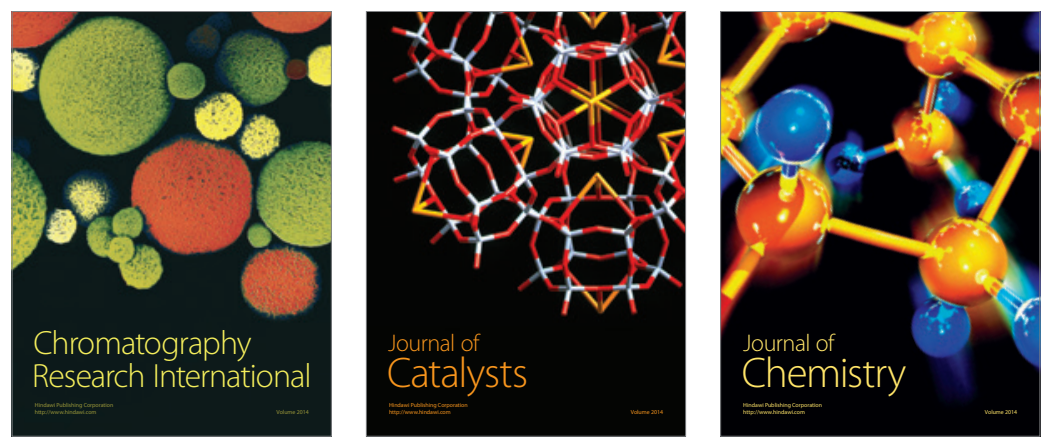
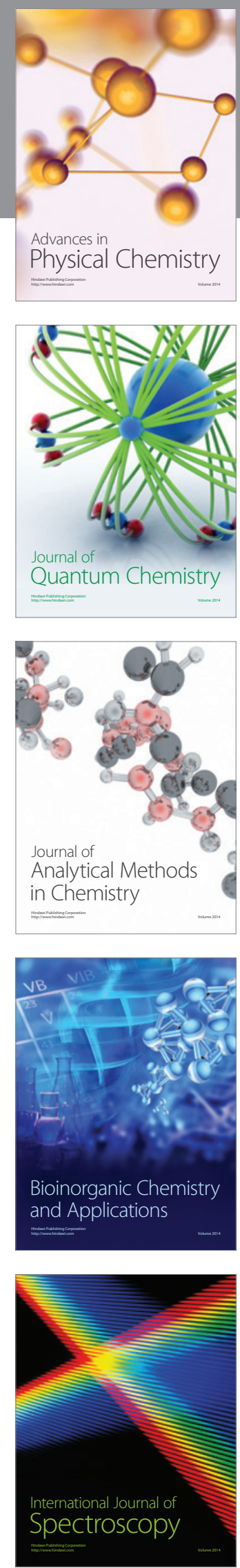